\title{
Comparative Evaluation of the Shear Bond Strength and Debonding Properties of a New Self Etch Adhesive Composite Cement and Self Etching Primer Composite System Used for Orthodontic Bracket bonding - An In Vitro Study
}

\section{Sameer Ahmad Malik ${ }^{1 *}$ and Laxmikanth $\mathbf{S M}^{2}$}

${ }^{1}$ Senior Lecturer, Department of Orthodontics, Oxford Dental College, Bangalore, India

${ }^{2} \mathrm{HOD}$, Department of Orthodontics, Oxford Dental College, Bangalore, India

*Corresponding Author: Sameer Ahmad Malik, Senior Lecturer, Department of Orthodontics, Oxford Dental College, Bangalore, India.
Received: March 02, 2021

Published: May 24, 2021

(C) All rights are reserved by Sameer Ahmad

Malik and Laxmikanth SM.

\section{Abstract}

Background of the Study: Over the last 50 years the bonding of various adhesives to enamel and dentin has developed a niche in nearly all areas of dentistry, including orthodontics. Combining conditioning and priming into a single step improves bonding time and cost-effectiveness to the clinician and Orthodontists use the acid-etch bonding technique to attach brackets to the enamel surface. Maintaining a sound, unblemished enamel surface after debonding the brackets is a primary concern to the clinician. Selfadhesive cements were introduced in 2002 as a new subgroup of resin cements. Self-adhesive cements do not require any pre treatment of the tooth surface. Once the cement is mixed, its application is a single clinical step. Sufficient research is not available with regard to whether self etching adhesives can be satisfactorily used in the field of orthodontics to bond the brackets. Maxcem Elite Chroma (Kerr Dental) is a self-etch/self-adhesive resin cement with a Colour Cleanup Indicator. The use of Maxcem Elite Chroma in the field of orthodontics is rather unexplored. Its basic properties such as SBS, the debonding properties and its effect on enamel demineralization need to be evaluated to commend its use for bonding in orthodontics.

Aims and Objective: Comparative evaluation of the shear bond strength and debonding properties of self etch adhesive composite cement (Maxcem Elite Croma) versus self etching primer composite system ((Transbond Plus SEP, 3M Unitek, Monrovia, Calif) used for orthodontic bracket bonding.

Materials and Methods: This was an in vitro study, conducted in controlled laboratory settings. Forty extracted human premolars extracted for purpose of orthodontic treatment. 20 premolars bonded with orthodontic brackets using the self-etch primer Transbond Plus (3M Unitek) containing both the acid and the primer by following manufacturer's recommendation and 20 premolars bonded with orthodontic brackets using the self-etching composite adhesive (Maxcem Elite ${ }^{\mathrm{TM}}$ Chroma Self-etch/Self-adhesive Resin Cement) cement by following manufacturer's recommendation). Results of continuous measurements were presented as mean \pm SD. Significance was assessed at 5\% level of significance at $95 \%$ confident interval. Mann Whitney U test was used to compare the shear bond strength of self-etching composite adhesive to self etch primer). Chi square test was used to evaluate the differences in ARI scores among both the groups. Chi square test showed that there were statistically significant differences among the groups (p $<0.001$ ).

Results: Maxcem elite chroma exhibited the highest mean shear bond strength of 25.7 MPa and Transbond Plus (3M Unitek, Monrovia, CA, USA) exhibited the mean shear bond strength of 18.7 MPA. Maxcem elite chroma exhibited the highest ARI index thereby demonstrating its better debonding properties.

Conclusion: Within the limitations of the current study we concluded that: 1. New self-etch, self-adhesive bonding system surprisingly showed high SBS as compared to self etch primer, 2. It also demonstrated a high ARI scores on the bracket surface which signifies that the debonding takes place mostly at tooth resin interface thereby reduces the chances of deleterious effects like crazing of enamel surface from debonding forces.

Keywords: Bonding; Shear Bond Strength; Adhesive Remanent Index; Self Etch Bonding Agent; Self Etch Adhesive Resin Cements 


\section{Introduction}

Over the last 50 years the bonding of various adhesives to enamel and dentin has developed a niche in nearly all areas of dentistry, including orthodontics.

Adhesion currently encompasses a combination of mechanical, adsorption, diffusion, and electrostatic phenomena [1]. Conventional adhesive systems use three different agents-an enamel conditioner, a primer solution, and an adhesive resin to bond orthodontic brackets to enamel.

A unique characteristic of some bonding systems in operative dentistry is that they combine the conditioning and priming agents into a single acidic primer solution for simultaneous use on both enamel and dentin [2,3]. Combining conditioning and priming into a single step improves bonding time and cost-effectiveness to the clinician and indirectly to the patient. These systems were used originally on dentin $[3,4]$. Essentially, the acidic part of the primer dissolves the smear layer and incorporates it into the mixture. Acidic primer solutions also demineralise the dentin and encapsulate the collagen fibres and hydroxyapatite crystals [5]. This simultaneous conditioning and priming allows penetration of the monomer into the dentin. The adhesive resin component will then diffuse into the primed dentin, producing a "hybrid layer [5]. While these conditioners were initially developed for use on dentin, studies have determined that adhesive systems combining conditioning and priming were also found to be effective when bonding to enamel [6].

Orthodontists use the acid-etch bonding technique to attach brackets to the enamel surface. Maintaining a sound, unblemished enamel surface after debonding the brackets is a primary concern to the clinician. As a result, bond failure at the bracket-adhesive interface or within the adhesive is more desirable (safer) than at the adhesive-enamel interface, because enamel fracture and crazing have been reported at bracket debonding especially with ceramic brackets [7]. It is possible that the depth of the etched enamel surface created by phosphoric acid may be a factor contributing to the incidence of enamel fracture [8-10]. Therefore, alternative enamel conditioners, such as maleic acid, and the newly introduced acidic primers that contain phenyl P may be beneficial, if they can maintain clinically useful orthodontic bracket bond strength while decreasing the depth of enamel dissolution.

Until recently, resin cements were divided into these two subgroups according to the adhesive system used to prepare the tooth prior to cementation. One group utilizes etch-and rinse adhesive in the other group, enamel and dentin are prepared using self-etching primers.

Self-adhesive cements were introduced in 2002 as a new subgroup of resin cements. These materials were designed with the intent to overcome some of the shortcomings of both conventional (zinc phosphate, polycarboxylate, and glass-ionomer cements) and resin cements, as well as to bring the favourable characteristics of different cement classes into a single product.

Self-adhesive cements do not require any pre treatment of the tooth surface. Once the cement is mixed, its application is a single clinical step, similar to the application procedures of zincphosphate and polycarboxylate cements. These are still relatively new and detailed information on their composition and adhesive properties is limited. Although the basic adhesion mechanism appears to be the same for all self-adhesive cements, its multifunctional monomers with phosphoric acid groups simultaneously demineralise and infiltrate enamel and dentin. The dominant setting reaction is the radical polymerization that can be initiated by light exposure or through the self-curing mechanism. As the smear layer is not removed, no postoperative sensitivity is expected. Self-adhesive cements are claimed to be moisture tolerant.

It has been demonstrated that shear bond strengths (SBSs) of brackets bonded using different "self-etch" primers (SEPs) were not significantly different from those associated with brackets bonded with the conventional acid-etch technique [9,11]. It has also been concluded that SEPs that produce a minimal etch pattern can still provide adequate bracket SBS [12]. Additionally, it was observed that when using a self-etching adhesive to bond brackets, no significant difference was seen when the SEP was cured either in separate steps or simultaneously with the bonding adhesive.

Sufficient research is not available with regard to whether self etching adhesives can be satisfactorily used in the field of orthodontics to bond the brackets. The SBS, the debonding properties and its effect on enamel demineralization have been documented rather elusively.

Maxcem Elite Chroma (Kerr Dental) is a self-etch/self-adhesive resin cement with a Colour Cleanup Indicator, to tell the optimal window of time to clean up excess cement. The use of Maxcem Elite Chroma in the field of orthodontics is rather unexplored. Its basic 
properties such as SBS, the debonding properties and its effect on enamel demineralization need to be evaluated to commend its use for bonding in orthodontics.

\section{Objectives of the Study}

The objectives of the current study are:

1. To evaluate the shear bond strengths (SBSs) of self-adhesive resin materials and compare them with self etch primer composite system.

2. To evaluate the ARI index of self-adhesive resin materials and compare them with self etch primer composite system.

\section{Materials and Methods}

This was an in vitro study, conducted in controlled laboratory settings. The materials used were:

1. Forty extracted human premolars extracted for purpose of orthodontic treatment

2. Thymol $0.1 \%$

3. Pumice

4. Polishing cups

5. Orthodontic premolar metal bracket (Ortho Organizers, MBT 0.022, Carlsbad, USA)

6. Self etch primer Transbond Plus (3M Unitek, Monrovia, CA, USA)

7. Self etching adhesive (Maxcem Elite ${ }^{\mathrm{TM}}$ Chroma Self-etch/Selfadhesive Resin Cement)

8. LED curing unit (Coltene S.P.E.C. ${ }^{\circledR}$ 3)

9. Plastic (PVC) rings for mounting premolars

10. Mounting jig

11. MecMesin (Intelligent Load Unit) Universal testing machine

12. Optical microscope (Olympus BX51).

Method of collection of data/methodology

In this study, the experimental model consisted of 1 control group and 1 experimental groups:

- Group 1: Control group: 20 premolars bonded with orthodontic brackets (Ortho Organizers, MBT 0.022, Carlsbad, USA) using the self-etch primer Transbond Plus (3M Unitek) containing both the acid and the primer by following manufacturer's recommendation.
- Group 2: Experimental group: 20 premolars bonded with orthodontic brackets (Ortho Organizers, MBT 0.022, Carlsbad, USA) using the self-etching composite adhesive (Maxcem Elite $^{\mathrm{TM}}$ Chroma Self-etch/Self-adhesive Resin Cement) cement by following manufacturer's recommendation.

Sample size estimation

Analysis: A priori: Compute required sample size

Input: Tail(s) = One

Effect size $\mathrm{d}=0.82$

$\alpha$ err prob $=0.05$

Power $(1-\beta$ err prob $)=0.80$

Allocation ratio $\mathrm{N} 2 / \mathrm{N} 1=1$.

Output: Noncentrality parameter $\delta=2.5930677$

Critical $t=1.6859545$

$\mathrm{Df}=38$

Sample size group $1=20$

Sample size group $2=20$

Total sample size $=40$

Actual power $=0.8163646$.

Methodology

Forty freshly extracted human premolars extracted for the purpose of orthodontic treatment were collected and stored in a solution of $0.1 \%$ (wt/vol) thymol.

\section{Inclusion criteria}

1. Extracted human premolar teeth extracted for the purpose of orthodontic treatment will be selected only if they have intact buccal enamel.

\section{Exclusion criteria}

2. Teeth having surface cracks from extraction forceps,

3. Teeth treated with chemical agents, and

4. Teeth having caries or restoration

5. Teeth undergone attrition.

6. Teeth previously not tested or bonded for any purposes.

Groups tested

Group 1 (control group)

Twenty premolar were bonded using the manufacturer's recommended protocol then is applied by continuously rubbing the self etch primer Transbond Plus (3M Unitek) on the enamel surface 
for 3 - 5 seconds. The SEP is then lightly dried using compressed air for 1 - 2 seconds. Each pre coated bracket was placed on the tooth and held for 10 seconds. The force gauge can be used to help assure a uniform adhesive thickness between the bracket and enamel. The bracket was then light cured using a LED curing light for 20 seconds (10 seconds for each proximal side).

Group 2 (experimental group)

Twenty premolars were bonded, following the manufacturer's recommendations, using the self-etchant adhesive (Maxcem
Elite $^{\mathrm{TM}}$ Chroma Self-etch/Self-adhesive Resin Cement). Maxcem Elite Chroma was applied on enamel surfaces, and bracket bases were bonded in place without separate enamel etching step before bonding. This self-etch, self adhesive resin cement was polymerized with LED curing light for $20 \mathrm{~s}$ each on both mesial and distal sides (i.e. 40 s total).

Compositions of adhesive materials used in this study

\begin{tabular}{|c|c|c|c|}
\hline Adhesive material & Manufacturer & Filler weight (\%) & Composition \\
\hline Transbond XT & $\begin{array}{c}\text { 3M Unitek, Monrovia, } \\
\text { CA, USA }\end{array}$ & 80 & $\begin{array}{c}\text { 35\% phosphoric acid, silane-treated quartz, bisphenol } \\
\text { A diglycidyl ether dimethacrylate, bisphenol A bis-(2- } \\
\text { hydroxyethyl)-ether dimethacrylate, silane-treated silica } \\
\text { self etch primer }\end{array}$ \\
\hline Maxcem Elite Chroma & $\begin{array}{c}\text { Kerr Italia, 84018 } \\
\text { Scafati, Italy }\end{array}$ & 69 & $\begin{array}{c}\text { GPDM, methacrylate ester monomers, HEMA, 4 methoxy- } \\
\text { phenol, cumene hydroperoxide, titanium dioxide and pig- } \\
\text { ments }\end{array}$ \\
\hline
\end{tabular}

GPDM: Glycerol Dimethacrylate Dihydrogen Phosphate; HEMA: 2-Hydroxyethyl Methacrylate; MEHQ: Monomethyl Ether Hydroquinone.

\section{Determination of Bond Strength and De bonding procedure}

Bond strength was determined by a universal testing machine (MecMesin Intelligent Load Unit) with $1 \mathrm{kN}$ load cell at crosshead speed of $1 \mathrm{~mm} /$ minute. Each tooth was oriented with the testing device as a guide such that its labial surface was parallel to the force during the shear strength test. An occlusal-gingival load was applied to the bracket with a blade-end steel rod attached to the crosshead of the universal testing machine. This produced a shear force at the bracket-tooth interface. The load at bracket failure was recorded electronically in Newtons by a computer connected to the MecMesin machine. The SBS values were calculated in megapascal by dividing the force by the area of the bracket base (MPa = N/ $\mathrm{mm}^{2}$ ). The maximum load necessary to debond or initiate bracket fracture was recorded in Newton and then converted into megapascal as a ratio of Newtons to the bracket surface area. The bracket surface area of Ortho Organizer maxillary firstpremolar bracket is $10.27 \mathrm{~mm}^{2}$.

\section{Adhesive remnant index}

After bond failure, the teeth and brackets were examined under 10x magnification. Any adhesive remaining after bracket removal was assessed using a modified Adhesive Remnant Index (ARI) and scored with respect to the amount of resin material adhering to the enamel surface. The ARI scale has a range between 5 and 1 :
Score $0=$ no adhesive left on bracket

Score $1=$ less than $25 \%$ of adhesive left on bracket

Score $2=25 \%$ of adhesive left on bracket

Score $3=50 \%$ of adhesive left on bracket

Score $4=75 \%$ of adhesive left on bracket

Score $5=100 \%$ of adhesive left on bracket.

\section{Results}

Shear bond strength

Mean SBS values (in MPa) of the two test groups and their descriptive statistics are shown in table 1 and figure 1 respectively. Maxcem elite chroma exhibited the highest mean shear bond strength of 25.7 MPa and Transbond Plus (3M Unitek, Monrovia, CA, USA) exhibited the mean shear bond strength of 18.7 MPA. The difference found between the two groups was seen to be statistically significant $(\mathrm{p}<0.005)$. Results of continuous measurements were presented as mean \pm SD. Significance was assessed at $5 \%$ level of significance at 95\% confident interval. Mann Whitney U test was used to compare the shear bond strength of self-etching composite adhesive to self etch primer.

\section{ARI Scores}

Table 2 shows ccomparison of ARI scores among the groups. Figure 2 shows the distributions of ARI scores, by percentage, for 
Comparative Evaluation of the Shear Bond Strength and Debonding Properties of a New Self Etch Adhesive Composite Cement and Self Etching Primer Composite System Used for Orthodontic Bracket bonding - An In Vitro Study

\begin{tabular}{|c|c|c|c|}
\hline & $\begin{array}{c}\text { Shear bond strength } \\
{[\text { Mean } \pm \text { SD] }}\end{array}$ & $\mathbf{Z}$ & $\mathbf{P}$ \\
\hline $\begin{array}{c}\text { Self-etching composite } \\
\text { adhesive } \\
\text { (Experimental) }\end{array}$ & $25.74 \pm 1.96$ & -5.458 & $0.00^{*}$ \\
\cline { 1 - 1 } $\begin{array}{c}\text { Self-etch primer (Con- } \\
\text { trol) }\end{array}$ & $18.77 \pm 1.64$ & \\
\hline \multicolumn{2}{|c|}{ Mann-Whitney U, *Statistically significant, $\mathrm{p}<0.05$} \\
\hline
\end{tabular}

Table 1: Comparison of shear bond strength of Self-etch primer and Self-etching composite adhesive.

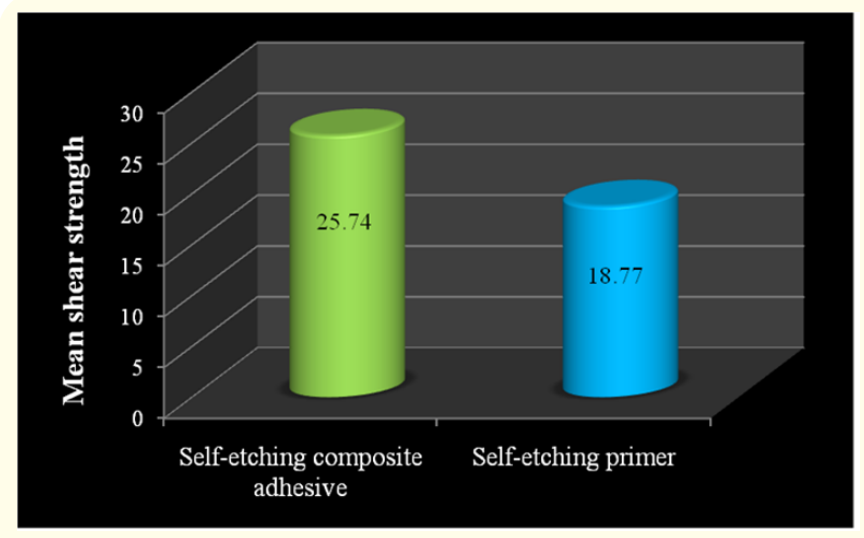

Figure 1: Comparison of shear bond strength of Self-etch primer and Self-etching composite adhesive. all the test groups. Chi square test showed that there were statistically significant differences among the groups ( $p<0.001)$. Chi square test was used to evaluate the differences in ARI scores among both the groups.

This table shows that the mean shear bond strength of self-etch primer (18.77 \pm 1.64 ) was less than self-etching composite adhesive $(25.74 \pm 1.96)$ and this difference was statistically significant $(\mathrm{p}=0.00)$.

This table shows that majority (30\%) of samples had score 3 followed by score $2(22.5 \%)$ and score 4 (20\%). In the experimental group, majority (35\%) of samples had score 3 followed by score $4(30 \%)$ and score 5 (20\%). In the control group, majority (35\%) of participants had score 2 followed by score 3 (25\%) and score 1 $(20 \%)$. However there was no significant difference between them $(\mathrm{p}=0.08)$.

\section{Discussion}

The direct bonding of orthodontic brackets has revolutionized and advanced the clinical practice of orthodontics. However, there is a need to improve the bonding procedure by saving time and minimizing enamel loss without jeopardizing a clinically useful bond strength. Although recent bonding systems have been proven

\begin{tabular}{|c|c|c|c|c|c|c|c|c|c|}
\hline \multirow{2}{*}{ Groups } & \multicolumn{6}{|c|}{ ARI scores (\%) } & \multirow[b]{2}{*}{ Total } & \multirow[b]{2}{*}{ Chi-square } & \multirow[b]{2}{*}{$\mathbf{P}$} \\
\hline & $\mathbf{0}$ & 1 & 2 & 3 & 4 & 5 & & & \\
\hline $\begin{array}{l}\text { Self-etching composite adhesive } \\
\text { (Experimental) }\end{array}$ & 0.0 & 2.5 & 5.0 & 17.5 & 15.0 & 10.0 & 50.0 & \multirow[t]{3}{*}{9.711} & \multirow[t]{3}{*}{0.08} \\
\hline Self-etch primer (Control) & 2.5 & 10.0 & 17.5 & 12.5 & 5.0 & 2.5 & 50.0 & & \\
\hline Total & 2.5 & 12.5 & 22.5 & 30.0 & 20.0 & 12.5 & 100 & & \\
\hline
\end{tabular}

Table 2: Comparison of ARI scores among the groups.

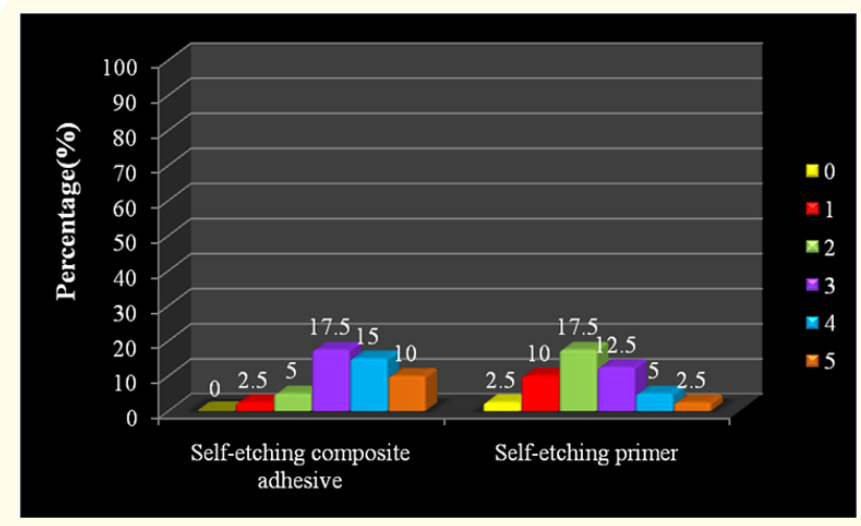

Figure 2: Comparison of API scores among the groups. reliable, improvements are still necessary to minimize technique sensitivity and reduce the chair time by decreasing the number of steps during the bonding procedure. Traditionally, using acid etchants followed by a primer was an essential part of the bonding procedure of composite adhesives, to allow good wetting and penetration of the sealant into the enamel surface $[10,11]$. The use of the new self adhesive resin cements for orthodontic purposes has not yet been fully evaluated. In general, they are thought to simplify the clinical handling of the adhesive systems by combining the etchant, primer and composite resin in 1 application. The present study evaluated 2 adhesive systems-Transbond Plus with a 2-component self-etch primer and Maxcem Elite Chroma self etching 
Comparative Evaluation of the Shear Bond Strength and Debonding Properties of a New Self Etch Adhesive Composite Cement and Self Etching Primer Composite System Used for Orthodontic Bracket bonding - An In Vitro Study

117

\begin{tabular}{|c|c|c|c|c|c|c|}
\hline Group & Score 0 (\%) & Score 1 (\%) & Score 2 (\%) & Score 3 (\%) & Score 4 (\%) & Score 5 (\%) \\
\hline TXT (Control) & $1(5)$ & $4(20)$ & $7(35)$ & $5(25)$ & $2(10)$ & $1(5)$ \\
\hline MEc (Experimental) & $0(0)$ & $1(5)$ & $2(10)$ & $7(35)$ & $6(30)$ & $4(20)$ \\
\hline
\end{tabular}

Table B

adhesive resin cement. The findings indicated that both bonding systems provided adequate shear bond strengths but Maxcem elite chroma exhibited the highest mean shear bond strength of 25.7 MPa and Transbond Plus exhibited the mean shear bond strength of 18.7 MPA. The difference found between the two groups was seen to be statistically significant $(\mathrm{p}<0.005)$. This was an in vitro study, and care should be taken in comparing the results with those that might be obtained in the oral environment. In addition, more research is needed to determine the shear bond strength of these new self-etch adhesive resin cements over a longer time period-e.g. 24 hours and 1 week after bonding and after thermocycling.

\section{Transbond ${ }^{\mathrm{TM}}$ plus self etching primer}

Minimum bond strength for clinically acceptable bracket bonding was reportedly 6 - $8 \mathrm{MPa}$ [12]. In the present study, Transbond XT self-etch resin materials showed clinically acceptable bond strengths for bracket bonding. It has been proved in many studies that the SBS of Transbond XT SEP is comparable to conventional Transbond XT. Transbond XT is a traditional composite resin filled with filler particles of diverse sizes [13] and which reportedly exhibited clinically acceptable bond strength values. Transbond XT was used as the gold standard in many SBS studies [12-42]. However, its SBS values varied widely between $8.9 \pm 3.9$ and $18.1 \pm 5.5$ $\mathrm{MPa}$ in literature. Mineral structures of the teeth used in these studies might have an effect on the SBS of different bonding agents, thus accounting for this large disparity in the SBS data of Transbond XT. Secondly, pressure exerted by the handpiece instrument during polishing might have an effect on tooth surface features, which would then affect the SBS of adhesive materials and cause differences in the SBS of Transbond XT among different studies.

\section{Phosphoric acid etchant versus self-etch adhesive systems}

Pre-treatment with $37 \%$ phosphoric acid increases bond strength $[43,44]$ because thick outer enamel layer may prevent the permeation of self-etch primers and bonding agents, thus leaving some areas partially unetched. This then results in formation of shorter and poorly defined resin tags. By removing the outer enamel with phosphoric acid etching, longer resin tags are formed and thus bond strength is increased [45]. On the other hand, selfetch primers and bonding agents have some advantages over the phosphoric acid etchant. Self-etch primers simplify the clinical handling of adhesive systems by combining the conditioning and priming agents into a single product ${ }^{4,5}$. Self-etch bonding agents prevent aggressive decalcification and bulk enamel loss which are characteristics of phosphoric acid etching [46]. This means that they reduce the risk of enamel damage due to their reduced ability to sufficiently etch and penetrate the enamel surface [47]. Most self-etch adhesives did not etch enamel as deeply as the phosphoric acid etchants did, but the shallow etching pattern compromised bonding to enamel [48]. Demineralization effects of self-etch primers and bonding agents are proportional to their acidity [49]. The lower the $\mathrm{pH}$, the higher the acidity, the deeper the etch; and the $\mathrm{pH}$ values of self-etch adhesive systems are higher than that of phosphoric acid etchant. Self-etch adhesive systems produce high bond strengths to human coronal dentin and ground enamel surfaces [50]. With enamel, phosphoric acid pre treatment improved the bond strength; with dentin, prior acid etching was detrimental to dentin bonding effectiveness and should be avoided [53,54]. Selfetch adhesives have high viscosity, which causes an incomplete resin decrease in its bracket retentive ability.

\section{Maxcem elite chroma}

Self-adhesive resin cements were developed to reduce the number of application steps and technique sensitivity associated with conventional resin cements. Maxcem Elite is a two-paste, dual-cure resin cement which combines the conditioning, priming, and adhesive agents into a single application. Maxcem Elite contains a proprietary redox initiator system: an efficient dual-cure mechanism that allows the resin to set quickly in the absence of light curing. Moreover, this proprietary redox initiator system eliminates the inherent discoloration of BPO/tertiary amine initiator systems for more esthetic restorations. However, when compared to conventional resin cements, self-adhesive resins reportedly provided inferior bonding to both enamel [52]. Maxcem Elite chroma has a colour indicator added to its predecessor Maxcem Elite. The colour indicator is pink when two solutions are mixed and as the material goes into initial set or gel phase, the pink colour fades away and turns colourless indicating optimal time to remove any excess cement. This property is particularly beneficial for bracket bonding in orthodontics, as it tells us the time to remove flash without disturbing the set bracket position significantly. After removing the flash the composite can be command cured by light cure devise. 
Comparative Evaluation of the Shear Bond Strength and Debonding Properties of a New Self Etch Adhesive Composite Cement and Self Etching Primer Composite System Used for Orthodontic Bracket bonding - An In Vitro Study

Bracket bond failure

Bond failure at bracket-adhesive interface, rather than at enamel-adhesive interface, is caused by the low flexural strength of composite resins. Brackets bonded to enamel surfaces are temporary: they shall be removed after active treatment. Therefore, the primary orthodontic goal is to maintain sound, unblemished enamel surfaces after debonding. Enamel damage can be caused by phosphoric acid etching, bracket removal or cleaning of the teeth after debonding. Phosphoric acid etching complicates the removal of residual adhesive on the enamel after debonding, subsequently leading to surface scratches, cracking, and loss of sound enamel [48].

ARI score "5" means that bond failure site is at adhesiveenamel interface, while score " 0 " means that detachment occurs at adhesive-bracket base interface. In the present study, statistically significant differences were found in the ARI scores among the groups. ARI scores of 3 and 4 were predominantly seen in Maxcem Elite. This meant that when phosphoric acid etching was omitted, interfacial bonding between enamel and adhesive became the weakest link. However, when these adhesive materials were applied to etched enamel with an SEP, the ARI decreased. From the perspective of cleaning up the teeth after debonding, it is advantageous and preferred for residual adhesive to remain at bracket bases. This makes cleaning of the enamel surfaces easier and faster, with less risk of causing damage to the enamel [51]. In the present study, Maxcem Elite yielded the highest SBS and higher incidences of ARI scores of 3 and 4 that is, a lesser amount of residual adhesive remained on the teeth. If adequate shear bond strength is attained, benefits of low ARI scores outweigh those of high ARI scores: detachment at enamel-adhesive interface lowers the risk of enamel damage and hence the probability of enamel cracks formation. But if both are achieved, that is, high SBS and high ARI like it was seen in maxcem elite group, it is highly desirable for an orthodontic adhesive. Hence it was demonstrated that Maxcem Elite chroma not only has an adequate SBS but also has a favourable debonding characteristics and that it can be considered as an alternative to total etch technique or self etch technique for bonding of brackets in orthodontics.

\section{Conclusion}

The purpose of this in vitro study was to evaluate of the shear bond strength and debonding properties of self etch adhesive composite cement (Maxcem Elite Croma) and self etching primer composite system ((Transbond Plus SEP, 3M Unitek, Monrovia, Calif) used for orthodontic bracket bonding. The self etch adhesive resin cements eliminates the need of separate etching and priming thereby reducing the operating time by a significant amount. Sufficient research is not available with regard to whether self etching adhesives can be satisfactorily used in the field of orthodontics to bond the brackets. The SBS, the debonding properties and its effect on enamel demineralization have been documented rather elusively.

Hence the current study was conducted to evaluate the same.

The conclusions from this study are as follows:

1. New self-etch, self-adhesive bonding system surprisingly showed high SBS as compared to self etch primer.

2. It also demonstrated a high ARI scores on the bracket surface which signifies that the debonding takes place mostly at tooth resin interface thereby reduces the chances of deleterious effects like crazing of enamel surface from debonding forces.

3. Both of the above properties are desirable for orthodontic bracket bonding and hence it can be considered as an alternative to traditional bonding systems.

Within the limitations of this in vitro study, we drew the above mentioned conclusion. However, further in vitro and in vivo studies of these self-etch, self adhesive bonding systems are needed to obtain data and findings useful and important for clinical practice which can be focussed to demonstrate following:

1. The effects of mineral structure of teeth and handpiece pressure on the SBS of different adhesive materials.

2. The effect of aging on the SBS of this new adhesive which can be checked for both in vivo and in vitro environments.

3. The surface characteristics of enamel before and after debonding when the self-etch, self adhesive bonding systems were used.

\section{Bibliography}

1. Summitt JB., et al. "Fundamentals of Operative Dentistry: A Contemporary Approach, 3rd ed. Hanover Park, Ill: Quintessence Publishing Co, Inc (2006): 184-210.

2. Bishara SE., et al. "Effect of an acidic primer on shear bond strength of orthodontic brackets". American Journal of Orthodontics and Dentofacial Orthopedics 114 (1998): 243-247.

3. Barkmeier WW and Erickson RL. "Shear bond strength of composite to enamel and dentin using Scotchbond multi-purpose". American Journal of Dentistry 4 (1994): 175-179. 
4. Bishara SE., et al. "Comparison of the shear bond strength of 2 self-etch primer/adhesive systems". American Journal of Orthodontics and Dentofacial Orthopedics 125 (2004): 348-350.

5. Bishara SE., et al. "Effect of a self-etch primer/adhesive on the shear bond strength of orthodontic brackets". American Journal of Orthodontics and Dentofacial Orthopedics 119 (2001): 621-624.

6. Vicente A., et al. "Shear bond strength of orthodontic brackets bonded with self-etching primers". American Journal of Dentistry 18 (2005): 246-260.

7. Goracci C., et al. "Microtensile bond strength of self-etching adhesive to enamel and dentin". The Journal of Adhesive Dentistry 6 (2004): 313-318.

8. Ostby AW., et al. "Effect of self-etchant pH on the shear bond strength of orthodontic brackets". American Journal of Orthodontics and Dentofacial Orthopedics (2008).

9. Bishara SE., et al. "The effect of modifying the self-etchant bonding protocol on the shear bond strength of orthodontic brackets". The Angle Orthodontist 77 (2007): 504-508.

10. Barkmeier WW and Erickson RL. "Shear bond strength of composite to enamel and dentin using Scotchbond multi-purpose". American Journal of Dentistry 7 (1994): 175-179.

11. Triolo PT Jr., et al. "Effects of etching time on enamelbond strengths". American Journal of Dentistry 6 (1993): 302-304.

12. Reynolds IR and Von Fraunhofer JA. "Direct bonding of orthodontic brackets - a comparative study of adhesives". British Journal of Orthodontics 3 (1976): 143-146.

13. Buonocore MG. "A simple method of increasing the adhesion of acrylic filling materials to enamel surfaces". Journal of Dental Research 34.6 (1955): 849-853.

14. Retief DH., et al. "Optimal concentration of phosphoric acid as an etching agent. Part III: Enamel wettability studies". Journal of Prosthetic Dentistry 53.1 (1985): 42-46.

15. Fox NA., et al. "A critique of bond strength testing in orthodontics". British Journal of Orthodontics 21.1 (1994): 33-43.
16. Bishara SE., et al. "Effect of a self-etch primer/adhesive on the shear bond strength of orthodontic brackets". American Journal of Orthodontics and Dentofacial Orthopedics 119.6 (2001): 621-624.

17. Bishara SE., et al. "Effect of an acidic primer on shear bond strength of orthodontic brackets". American Journal of Orthodontics and Dentofacial Orthopedics 114.3 (1998): 243-247.

18. Bishara SE., et al. "The effect of repeated bonding on the shear bond strength of different orthodontic adhesives". American Journal of Orthodontics and Dentofacial Orthopedics 121.5 (2002): 521-525.

19. Cal-Neto JP., et al. "Effect of a self-etching primer on shear bond strength of adhesive precoated brackets in vivo". The Angle Orthodontist 76.1 (2006): 127-131.

20. Romano FL., et al. "Shear bond strength of metallic orthodontic brackets bonded to enamel prepared with self-etching primer". The Angle Orthodontist 75.5 (2005): 849-853.

21. Vilchis RJ., et al. "Shear bond strength of orthodontic brackets bonded with different self-etching adhesives". American Journal of Orthodontics and Dentofacial Orthopedics 136.3 (2009): 425-430.

22. Cehreli ZC., et al. "Effect of self-etching primer and adhesive formulations on the shear bond strength of orthodontic brackets". American Journal of Orthodontics and Dentofacial Orthopedics 127.5 (2005): 573-579.

23. Amra I., et al. "Xeno III self-etching adhesive in orthodontic bonding: the next generation". American Journal of Orthodontics and Dentofacial Orthopedics 131.2 (2007): 160.

24. Bishara SE., et al. "Early shear bond strength of a one-step self-adhesive on orthodontic brackets". The Angle Orthodontist 76.4 (2006): 689-693.

25. Bishara SE., et al. "Early shear bond strength of a one-step self-adhesive on orthodontic brackets". The Angle Orthodontist 76.4 (2006): 689-693.

26. Abo-Hamar SE., et al. "Bond strength of a new universal selfadhesive resin luting cement to dentin and enamel". Clinical Oral Investigations 9.3 (2005): 161-167. 
27. De Munck J., et al. "Bonding of an auto-adhesive luting material to enamel and dentin". Dental Materials 20.10 (2004): 963971.

28. Minick GT., et al. "Bracket bond strengths of new adhesive systems". American Journal of Orthodontics and Dentofacial Orthopedics 135.6 (2009): 771-776.

29. Vicente A., et al. "A comparison of the shear bond strength of a resin cement and two orthodontic resin adhesive systems". The Angle Orthodontist 75.1 (2005): 109-113.

30. Lührs AK., et al. "Shear bond strength of self-etch adhesives to enamel with additional phosphoric acid etching". Operative Dentistry 33.2 (2008): 155-162.

31. Walter R., et al. "Enamel and dentin bond strengths of a new self-etch adhesive system". Journal of Esthetic and Restorative Dentistry 23.6 (2011): 390-396.

32. Radovic I., et al. "Self-adhesive resin cements: a literature review". Journal of Adhesive Dentistry 10.4 (2008).

33. Ferracane JL., et al. "Self-adhesive resin cements-chemistry, properties and clinical considerations". Journal of Oral Rehabilitation 38.4 (2011): 295-314.

34. Fleming PS., et al. "Self-etch primers and conventional acidetch technique for orthodontic bonding: a systematic review and meta-analysis". American Journal of Orthodontics and Dentofacial Orthopedics 142.1 (2012): 83-94.

35. Nakamura T., et al. "Mechanical properties of new self-adhesive resin-based cement". Journal of Prosthodontic Research 54.2 (2010): 59-64.

36. Chu CH., et al. "Orthodontic bonding with self-etching primer and self-adhesive systems". The European Journal of Orthodontics 33.3 (2010): 276-281.

37. Al-Suleiman M., et al. "Mechanical Evaluation of the Effect of Reducing Phosphoric Acid Concentrations and Etching Duration on the Bond Strength of Orthodontic Brackets". Journal of Dental Health, Oral Disorders and Therapy 2.2 (2014): 1-5.

38. Alkis H., et al. "Microleakage under orthodontic brackets bonded with different adhesive systems". European Journal of Dentistry 9.1 (2015): 117.
39. Hellak A., et al. "Shear bond strength of three orthodontic bonding systems on enamel and restorative materials". BioMed Research International (2016).

40. Hattar S., et al. "Bond strength of self-adhesive resin cements to tooth structure". The Saudi Dental Journal 27.2 (2015): 7074.

41. Ongkowidjaja F., et al. "A comparison of orthodontic bracket shear bond strength on enamel deproteinized by $5.25 \%$ sodium hypochlorite using total etch and self-etch primer". In Journal of Physics: Conference Series 884.1 (2017): 012083.

42. Scougall-Vilchis RJ., et al. "Examination of six orthodontic adhesives with electron microscopy, hardness tester and energy dispersive X-ray microanalyzer". The Angle Orthodontist 78 (2008): 655-661.

43. Scougall Vilchis RJ., et al. "Effects of 6self-etching primers on shear bond strength of orthodontic brackets". American Journal of Orthodontics and Dentofacial Orthopedics 135 (2009): e1-7.

44. Scougall Vilchis RJ., et al. "Shear bond strength of orthodontic brackets bonded withdifferent self-etching adhesives". American Journal of Orthodontics and Dentofacial Orthopedics 136 (2009): 425-430.

45. Kanemura N., et al. "Tensile bond strength to and SEM evaluation of ground and intact enamel surfaces". Journal of Dentistry 27 (1999): 523-530.

46. Arhun N., et al. "Shear bond strength of orthodontic brackets with 3 self-etch adhesives". American Journal of Orthodontics and Dentofacial Orthopedics 129 (2006): 547-550.

47. Eminkahyagil N., et al. "Shear bond strength of orthodontic brackets with newly developed antibacterial self-etch adhesive". The Angle Orthodontist 75 (2005): 843- 848.

48. Kim MJ., et al. "Phosphoric acid incorporated with acidulated phosphate fluoride gel etchant effects on bracket bonding". The Angle Orthodontist 75 (2005): 678-684.

49. Di Francescantonio M., et al. "Bond strength evaluation of selfetch and total-etch adhesive systems on intact and ground human enamel”. Brazilian Journal of Oral Sciences 6 (2007): $1462-1466$. 
50. Kiremitci A., et al. "Bonding to enamel and dentin using selfetching adhesive systems". QI. Quintessence International 35 (2004): 367-370.

51. Usumez S., et al. "Effect of lightemitting diode on bond strength of orthodontic brackets". The Angle Orthodontist 74 (2004): 259-263.

52. Işman E., et al. "Inadequate shear bond strengths of self-etch, self-adhesive systems for secure orthodontic bonding". Dental Materials Journal 31.6 (2012): 947-953.

53. De Munck J., et al. "Bonding of an auto-adhesive luting material to enamel and dentin”. Dental Materials 20 (2004): 963-971.

54. Yang B., et al. "Micro-tensile bond strength of three luting resins to human regional dentin". Dental Materials 22 (2006): 4556.

Volume 5 Issue 6 June 2021

(C) All rights are reserved by Sameer Ahmad Malik and Laxmikanth SM. 